\title{
ASO Author Reflections: How Do We Improve Ergonomics for the Breast Surgeon During Nipple-Sparing Mastectomies?
}

\author{
Katherine Kopkash, $\mathrm{MD}^{1,2}$ \\ ${ }^{1}$ NorthShore University HealthSystem, Breast Surgery, Evanston, IL; ${ }^{2}$ University of Chicago Pritzker School of Medicine, \\ Chicago, IL
}

\section{PAST}

We spend thousands of hours training to become a breast surgical oncologist. The focus of this extensive process was caring for a patient throughout the surgical journey. But what about caring for the surgeon?

Nipple-sparing mastectomies (NSM) are an extremely demanding procedure for the surgeon. ${ }^{1-3}$ The mental and physical workload for these surgeries is significant. As the nipple-sparing mastectomy procedure becomes more prevalent, ${ }^{4}$ we cannot help but wonder the toll this will take on the surgeon.

\section{PRESENT}

Our group initially studied the ergonomic workload for NSM using electromyography (EMG) analysis of the upper trapezii, cervical erector spinae, anterior deltoid, and lumbar erector spinae muscle groups and a questionnaire. ${ }^{1}$ We then got to the crucial question: how do we improve it? We implemented a kinesiologist-created exercise program and an operating room positioning protocol (EOPP) and then studied its effects, again using EMG analysis and a questionnaire.

We saw a significant decrease in muscle workload of the bilateral upper trapezii muscle groups after the EOPP was implemented. However, this decrease in muscle workload did not seem to make the surgery any easier for the

(C) Society of Surgical Oncology 2021

First Received: 1 July 2021

Accepted: 1 July 2021

Published Online: 12 August 2021

K. Kopkash, MD

e-mail:kkopkash@northshore.org surgeon, with the surgeons reporting the procedures to be more mentally and physically demanding following EOPP. ${ }^{5}$

\section{FUTURE}

We are now aware of the difficulties associated with NSM and our focus has shifted to how do we improve. I believe we can take better care of our surgeons who perform NSM by maximizing their ergonomics. I am excited to see how this can be accomplished, possibly by improving instrumentation, specifically our retractors, and trying alternative methods, such as the robotic approach.

\section{REFERENCES}

1. Kopkash K, Novak K, Kuchta K, et al. The "nipple whipple"?! A pilot study to assess the ergonomic effects of nipple-sparing mastectomy. Ann Surg Oncol. 2019;26(10):3216-23.

2. Hallbeck MS, Law KE, Lowndes BR, et al. Workload differentiates breast surgical procedures: NSM associated with higher workload demand than SSM. Ann Surg Oncol. 2020;27(5):1318-26.

3. Jackson RS, Sanders T, Park A, et al. Prospective study comparing surgeons' pain and fatigue associated with nipple-sparing versus skin-sparing mastectomy. Ann Surg Oncol. 2017;24(10):3024-31.

4. Valero MG, Moo TA, Muhsen S, et al. Use of bilateral prophylactic nipple-sparing mastectomy in patients with high risk of breast cancer. Br J Surg. 2020;107(10):1307-12.

5. Kopkash K, Novak K, Murphy R, et al. Improving the breast surgeon's ergonomic workload for nipple-sparing mastectomies using exercise and an operating room positioning protocol. Ann Surg Oncol. 2021. https://doi.org/10.1245/s10434-021-10447-7.

Publisher's Note Springer Nature remains neutral with regard to jurisdictional claims in published maps and institutional affiliations. 